\title{
Health-related quality of life in patients with recurrent pericarditis: results from a phase 2 study of rilonacept
}

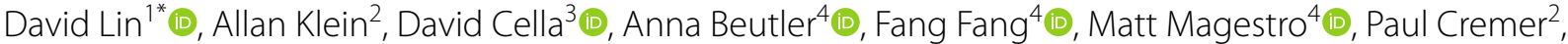

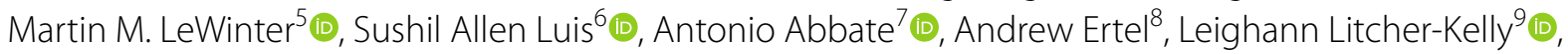 \\ Brittany Klooster ${ }^{9} \mathbb{D}$ and John F. Paolini $i^{4}$
}

\begin{abstract}
Background: Impact of recurrent pericarditis (RP) on patient health-related quality of life (HRQoL) was evaluated through qualitative patient interviews and as an exploratory endpoint in a Phase 2 trial evaluating the efficacy and safety of rilonacept (IL-1 a/IL-1 $\beta$ cytokine trap) to treat RP.

Methods: Qualitative interviews were conducted with ten adults with RP to understand symptoms and HRQoL impacts, and the 10-item Patient-Reported Outcomes Measurement Information System Global Health (PROMIS GH) v1.2 was evaluated to determine questionnaire coverage of patient experience. The Phase 2 trial enrolled participants with active symptomatic RP (A-RP, $n=16)$ and corticosteroid-dependent participants with no active recurrence at baseline (CSD-RP, $n=9)$. All participants received rilonacept weekly during a 6-week base treatment period (TP) plus an optional 18-week extension period (EP). Tapering of concomitant medications, including corticosteroids (CS), was permitted during EP. HRQOL was assessed using the PROMIS GH, and patient-reported pain and blood levels of c-reactive protein (CRP) were collected at Baseline and follow-up periods. A secondary, descriptive analysis of the Phase 2 trial efficacy results was completed using HRQoL measures to characterize both the impact of RP and the treatment effect of rilonacept.

Results: Information from qualitative interviews demonstrated that PROMIS GH concepts are relevant to adults with RP. From the Phase 2 trial, both participant groups showed impacted HRQoL at Baseline (mean PROMIS Global Physical Health [GPH] and Global Mental Health [GMH], were lower than population norm average). In A-RP, GPH/MPH improved by end of base TP and were sustained through EP (similar trends were observed for pain and CRP). Similarly, in CSD-RP, GPH/MPH improved by end of TP and further improved during EP, during CS tapering or discontinuation, without disease recurrence (low pain scores and CRP levels continued during the TP and EP).
\end{abstract}

Conclusion: This is the first study demonstrating impaired HRQoL in RP. Rilonacept treatment was associated with HRQoL improvements using PROMIS GH scores. Maintained/improved HRQoL during tapering/withdrawal of CS without recurrence suggests that rilonacept may provide an alternative to CS.

Trial registration: ClinicalTrials.Gov; NCT03980522; 5 June 2019, retrospectively registered; https://clinicaltrials.gov/ct2/ show/NCT03980522.

\footnotetext{
*Correspondence: David.Lin@allina.com

${ }^{1}$ Abbott Northwestern's Heart Hospital, Minneapolis Heart Institute, 800

East 28th Street, 2nd Floor, Minneapolis, MN 55407, USA

Full list of author information is available at the end of the article
}

(c) The Author(s) 2021. This article is licensed under a Creative Commons Attribution 4.0 International License, which permits use, sharing, adaptation, distribution and reproduction in any medium or format, as long as you give appropriate credit to the original author(s) and the source, provide a link to the Creative Commons licence, and indicate if changes were made. The images or other third party material in this article are included in the article's Creative Commons licence, unless indicated otherwise in a credit line to the material. If material is not included in the article's Creative Commons licence and your intended use is not permitted by statutory regulation or exceeds the permitted use, you will need to obtain permission directly from the copyright holder. To view a copy of this licence, visit http://creativecommons.org/licenses/by/4.0/. The Creative Commons Public Domain Dedication waiver (http://creativecommons.org/publicdomain/zero/1.0/) applies to the data made available in this article, unless otherwise stated in a credit line to the data. 
Keywords: Pericarditis, Interleukin-1 cytokine trap, Health-related quality of life, Recurrent pericarditis

\section{Introduction}

Pericarditis, or inflammation of the pericardium, has a variety of etiologies but is most commonly referred to as "idiopathic" $[1,2]$. The primary symptom of pericarditis is debilitating chest pain. Pericarditis is considered recurrent if symptoms and inflammation recur at least 4 weeks after an initial acute episode [1]. Recurrent pericarditis (RP) affects approximately $15-30 \%$ of patients who have an acute episode of pericarditis, and up to $50 \%$ of patients who experience one recurrence will experience two or more [1]. Empiric "off-label" therapy with nonsteroidal anti-inflammatory drugs [NSAIDs] and colchicine is often used successfully to treat the first pericarditis episode or initial recurrence [3]. Treatment options for patients with multiple recurrences, however, are limited, and there is a high unmet medical need for patients who have inadequate response (i.e., continued recurrence or incomplete symptom resolution) to, who cannot tolerate, standard therapy [1] or who have persistent underlying disease. Given that the cytokines interleukin-1 alpha (IL$1 \alpha)$ and beta (IL-1 $\beta)$ are implicated in RP etiology $[2,4]$, rilonacept, an IL- $1 \alpha$ and IL- $1 \beta$ cytokine trap, was evaluated in clinical trials for the treatment of RP $[5,6]$ and is now the first treatment approved by FDA for RP.

While the impact of RP on patients' health-related quality of life (HRQoL) has been reported in the literature [7-12] and is thought to be due to the primary symptom of the condition (e.g., chest pain) and the resulting uncertainty and anxiety about new recurrences, impact on HRQoL has not been explicitly evaluated in previous clinical research. In addition, corticosteroids (CS), despite well-known warnings and precautions in patients with cardio-metabolic comorbidities, are widely used to treat RP [13], putting patients at risk for additional adverse events, including recurrence and steroid dependence [14]; comorbidities associated with chronic CS use may also lead to adverse impacts on HRQoL [15, 16].

Both qualitative and quantitative research approaches were used to explore the HRQoL impacts experienced by adults with RP and how those impacts may change in response to treatment. Qualitative interviews were conducted with ten adults with RP to document the patient experience of RP symptoms and HRQoL impacts (known as concept elicitation interviews). Results from these interviews were used to develop a conceptual model of RP. In addition, a Phase 2 clinical trial of rilonacept for the treatment of RP included an HRQoL patientreported outcome (PRO) questionnaire as an exploratory endpoint. Therefore, the objective of these two streams of research is to evaluate HRQoL in patients with RP: specifically, to confirm whether the concepts assessed with the HRQoL PRO questionnaire used in the Phase 2 clinical trial are relevant to patients with RP (based on patient reports during the qualitative interviews) and to evaluate the effect of rilonacept treatment on physical and mental aspects of HRQoL.

\section{Methods}

The sections below describe the methodology used for both the qualitative interviews with patients and the clinical trial study design relevant to the current research objective.

\section{Methods for qualitative patient interviews to develop a patient-centric conceptual model}

To understand the patient experience of RP, one-on-one telephone interviews were conducted with adults with a confirmed diagnosis of RP.

\section{Participants for qualitative interviews}

The qualitative interview study was approved by a centralized independent review board (IRB); following approval, potentially eligible participants were identified from clinical sites through review of medical records. Key inclusion criteria for the qualitative study included: age 18 years or older and a clinical diagnosis of RP (either idiopathic or due to post-pericardiotomy syndrome, adult onset Still's Disease, or Dressler's Syndrome), defined as the first episode of acute pericarditis (as defined by the 2015 European Society of Cardiology Guidelines for the Diagnosis and Management of Pericardial Diseases) [1] followed by at least one pericarditis recurrence after a symptom-free interval of at least 4-6 weeks. Key exclusion criteria for the study included: individual was currently enrolled in another clinical interventional study for $\mathrm{RP}$; individual had a diagnosis of RP that was secondary to specific prohibited etiologies, including tuberculosis, neoplastic, purulent, or radiation, post-thoracic blunt trauma, myocarditis, or systemic autoimmune diseases (with the exception of adult onset Still's Disease).

Potentially eligible participants were presented with study information by a recruiting clinician (or his/her representative), and once participants provided a signed informed consent form, the clinical site completed a screening document to determine participants' eligibility. Participant interviews were scheduled once eligibility was confirmed. 


\section{Interview conduct}

One-on-one, 60-min telephone interviews with ten adults aged 18-75 years with RP were conducted. Interviewers used a semi-structured interview guide to facilitate a conversational-style interview and included open-ended questions to understand the patient experience of RP and its treatments, specifically what signs, symptoms, and HRQoL impacts are experienced in relation to RP from the patient perspective.

Interview guide questions included:

- "Could you please start by telling me about the first signs or symptoms of [participant's term for RP] you noticed?

- "Does the [patient-reported sign/symptom] have any impact on your daily life? If so, how?"

- "Have there been any changes to your daily life because of [participant's term for RP]? If so, can you please describe?"

\section{Data handing and analysis}

Interviews were audio-recorded after obtaining participant consent, transcribed, and anonymized. These transcripts were coded and qualitatively analyzed using the ATLAS.ti software program (ATLAS.ti Scientific Software Development GmbH, Berlin, Germany). The goal of transcript coding was to organize and catalog participants' descriptions of the characteristics of RP, in order to develop a patient-centric conceptual model of RP signs, symptoms, and impact concepts. A conceptual model is a heuristic classification scheme that links a specified disease state or condition to its proximal and increasingly distal health outcomes [17], acts as a framework for understanding a disease and/or its treatment, specifies the potentially relevant outcomes for a program of research, and informs the selection of measurement concepts to foster the development of questionnaires, outcomes, and endpoints. Specifically, to characterize the specific applicability of the Patient Reported Outcome Measurement Information System Global Health (PROMIS GH v1.2) [17] questionnaire (described below) for capturing the HRQoL impacts experienced by patients with RP, conceptual mapping was conducted to compare the concepts within the conceptual model against PROMIS GH v1.2 individual items.

\section{Methods for the Phase 2 study KPL-914-C001}

The methodology of the Phase 2 clinical trial of rilonacept for the treatment of RP (clinicaltrial.gov: NCT03980522) is provided in detail by Klein and colleagues [5]. To summarize, this was a multicenter, open-label, single-activearm Phase 2 study that enrolled two specific patient populations of adults with RP: (1) patients with an active recurrence who were symptomatic and had signs of inflammation at baseline (A-RP) and (2) patients who were not having an active recurrence but were dependent on CS (CSD-RP). All participants received weekly subcutaneous (SC) injections of rilonacept for 6 weeks during the treatment period (TP) and were invited to continue weekly SC injections for up to 18 weeks in the extension period (EP).

\section{Participants}

Adults (18-75 years of age) with RP (idiopathic or postpericardiotomy syndrome etiology) were enrolled and stratified into one of two participant groups, A-RP and CSD-RP. Participants in the A-RP group either (1) had evidence of elevated c-reactive protein (CRP) at baseline or (2) did not have elevated CRP, potentially due to concomitant medications (such as CS) but had evidence of pericardial inflammation by cardiac magnetic resonance imaging. Participants in the CSD-RP group had CS-dependent disease (based on information from the investigator regarding prior recurrences when taking medication) and did not have active pericarditis symptomatology or elevated CRP at baseline.

\section{Assessments}

Blood levels of CRP were evaluated weekly in TP, and then monthly, to measure inflammation from baseline to the end of the EP for all participants. In addition, two PRO questionnaires were completed by all participants during the clinical trial. They included:

1. A single-item 11-point numeric rating scale (NRS) for average pericarditis pain intensity with a 24-h recall window (with $0=$ no pain to $10=$ pain as bad as it could be) [18-20] was completed weekly in TP and monthly in EP from baseline to the end of the EP.

2. The 10-item PROMIS GH v1.2 questionnaire was also completed by participants at up to five timepoints during the clinical trial to assess HRQoL [21]. The analyses presented here focused on the following three most critical timepoints: baseline (Day 0), end of TP (Week 6), and Final Visit (end of EP). Items 1-7 ask participants to think about their general health and are rated on a five-point response scale (with higher scores associated with better quality of life). Items 8-10 ask participants to report on the emotional problems, fatigue, and pain over the last seven days, with Items 8 and 9 rated on a five-point response scale (with higher scores associated with better quality of life) and Item 10 rated on a $0-10$ NRS (with higher scores associated with more pain). Two domain scores are created from the 10-item 
scale, the global physical health (GPH) and the global mental health $(\mathrm{GMH})$. The GPH is scored by averaging together the global03 (physical health), global06 (physical function), global07 (pain) and global08 (fatigue) items. The GMH is scored by averaging together the global02 (quality of life), global04 (mental health), global05 (satisfaction with discretionary social activities), and global10 (emotional problems) [21]. The published US-generalized normative scores for both of these domains are a mean score of 50 and a standard deviation (SD) of 10 [21].

\section{Procedure and analyses}

All participants received rilonacept SC injections weekly for 6 weeks until the end of TP and were invited to continue weekly SC injections (at the same dose) during an optional 18-week EP. For those on other concomitant medications for RP at baseline (participants in both the A-RP and CSD-RP groups), including CS, the option to taper was offered during the EP. Participants completed the PRO questionnaires (PROMIS and Pain NRS) at study visits, including telephone and site visits. Blood levels for CRP were also assessed during clinical site visits or via visiting nurse or local contract laboratory; CRP was analysed via a central laboratory.

The analyses presented in the results are descriptive, given the small sample size of the clinical trial and the single-active-arm design. Specifically, results are reported as means and $\mathrm{SD}$, with ranges of values for each participant group. While participants were asked to complete the HRQoL questionnaire at multiple timepoints in the clinical trial, the analyses focus on the baseline, (Day 0), end of base TP (Week 6), and Final Visit at end of EP timepoints. Effect sizes (ESs; Cohen's d) were calculated with 95\% confidence intervals (CI) to evaluate the magnitude of the change from baseline to end of EP for each patient group for the HRQoL scores. ESs $\geq 0.80$ were considered large; $\geq 0.50$ to $<0.80$, medium; and $\geq 0.2$ to $<0.5$, small [22]. In addition, the descriptive analyses also include the weekly pericardial pain NRS scores and CRP blood levels that were collected at study visits.

\section{Results}

\section{Qualitative patient interviews}

Qualitative interviews were conducted via telephone with ten adults diagnosed with RP to understand the patient experience of the condition, including the signs, symptoms, and HRQoL impacts. Participants were recruited from three clinical sites in the US. The mean age of the participants was 58.5 years $(\mathrm{SD}=11.5)$, and six participants $(60.0 \%)$ were female. Clinicians reported that participants exhibited the following RP types: idiopathic $(\mathrm{n}=4,40.0 \%)$, post-pericardiotomy syndrome $(\mathrm{n}=4$, $40.0 \%)$, adult-onset Still's Disease $(n=1,10.0 \%)$, and Dressler's syndrome $(n=1,10.0 \%)$. The majority of participants reported taking over-the-counter or prescription anti-inflammatory medications $(n=7,70.0 \%)$, and half reported that they had previously taken CS $(n=5$, $50.0 \%)$ for their RP.

A total of 13 symptoms and 34 impacts across 11 domains were reported by participants during these qualitative interviews and are summarized in Table 1 and were organized into a conceptual model (Fig. 1). All participants reported experiencing chest pain $(n=10$, $100.0 \%)$, with seven $(n=7,70.0 \%)$ stating it is the most bothersome symptom, and five $(\mathrm{n}=5,50.0 \%)$ reporting it is the most important symptom to improve. After chest pain, the next most frequently reported signs or symptoms (reported by at least half of the participants) were tiredness $(n=8,80.0 \%)$, shortness of breath $(n=7$, $70.0 \%)$, fever $(n=6,60.0 \%)$, and heart palpitations $(n=5$, $50.0 \%$ ). The most frequently reported impacts (reported by at least half of the participants) were inability to exercise $(n=8,80.0 \%)$, disrupted sleep $(n=7,70.0 \%)$, fear $(\mathrm{n}=6,60.0 \%)$, inability to go to social events $(n=6$, $60.0 \%)$, interruption of daily activities $(n=6,60.0 \%)$, absenteeism $(\mathrm{n}=5,50.0 \%)$, and impaired ability to do housework $(\mathrm{n}=5,50.0 \%)$.

In order to confirm that the assessment of HRQoL completed by participants in the Phase 2 clinical trial captured concepts relevant to adults with RP, concepts reported during the qualitative interviews were mapped to the ten items of the PROMIS GH v1.2 questionnaire. Table 2 shows the results of this exercise, with representative patient quotes from the qualitative interviews for each of the items of the PROMIS GH questionnaire. In particular, adults reported symptoms and HRQoL impacts during the interviews that are included in the PROMIS GH questionnaire, such as pain, social and emotional impacts, and physical functioning.

\section{Phase 2 study rilonacept}

Twenty-five participants were enrolled in a multicenter, open-label, single-active-arm Phase 2 clinical trial of rilonacept, with an average age of $42.8 \pm 10.5$ years ( \pm indicates SD; range 26-62); most were female $(n=15$, $60.0 \%)$ and white $(n=22,88.0 \%)$. Participants had a mean number of prior recurrences of 2.6 (range 1-8), average duration of disease of $2.2 \pm 1.9$ years (range $0.2-$ 7.9 years), and average number of pericarditis episodes per year of $3.9 \pm 3.7$ (range 0.54-15). Based on their baseline symptoms and signs of pericardial inflammation, there were two groups of participants: those experiencing an active recurrence who were symptomatic with evidence of inflammation (A-RP; $n=16)$, and those 
Table 1 Patient-reported recurrent pericarditis symptom and impact domains description table

\begin{tabular}{|c|c|c|}
\hline \multirow[t]{3}{*}{$\begin{array}{l}\text { Symptom or impact domain } \\
\text { reported by participant }\end{array}$} & Description $^{a}$ & $\begin{array}{l}\text { Frequency of } \\
\text { participant } \\
\text { reports }^{\text {b }}\end{array}$ \\
\hline & & $(N=10)$ \\
\hline & & n (\%) \\
\hline
\end{tabular}

Symptoms

Chest pain

Tiredness

Shortness of breath

Fever

Heart palpitations

Chest pressure

Cough

Swelling

Abdominal pain

Bone pain

Difference in breathing

Flutters

Neck pain

Impact domains

Activities of daily living

Physical impacts

Psychological impacts

Sleep impacts

Social impacts

Relationship impacts

Work or school impacts
Described as sharp, stabbing, dull, or aching pain or pressure in the chest, which can radiate to the neck and shoulders

Described as physical exhaustion lasting a few days, which may co-occur with shortness of breath and affect one's activity level

Described as difficulty breathing and losing breath quickly, similar to a feeling of suffocation

$0(100.0 \%)$

$8(80.0 \%)$

$7(70.0 \%)$

Described as a low-grade fever that can include hot flashes or chills

$6(60.0 \%)$

$5(50.0 \%)$

$3(30.0 \%)$

Described as discomfort or heaviness in the chest

$3(30.0 \%)$

Described as uncomfortable and painful sporadic coughing episodes triggered by a tickling feeling

$2(20.0 \%)$

$1(10.0 \%)$

$1(10.0 \%)$

$1(10.0 \%)$

Described as sharp nerve pain in the neck affecting neck mobility

$9(90.0 \%)$

Described in the following ways

Inability to complete plans and daily activities

Inability to begin or complete household tasks, such as cleaning, cooking, and/or yard work

Impacts on driving (or fear of driving due to symptoms)

Diet and lifestyle changes

Inability to go shopping

Described in the following ways

$8(80.0 \%)$

Difficulty exercising and restrictions on exercising

Feeling dizzy (due to shortness of breath)

Inability to lay down (due to pain and shortness of breath)

Feeling the need to rest (due to heart palpitations)

Described in the following ways

Feeling scared because of symptoms

Feeling depressed because of symptoms

Feeling anxious, worried, or concerned because of chest pain

Not feeling normal and wanting to feel normal (due to symptoms)

Annoyance due to symptoms

Feeling like a burden to others

Feeling miserable due to chest pain

Described in the following ways

Waking up frequently or suddenly after falling asleep (associated with chest pain and shortness of breath)

$7(70.0 \%)$

$6(60.0 \%)$

$6(60.0 \%)$

Described in the following ways

Emotional distance or less activity with significant other

Feeling distant from family

Not being able to support family as much

Described in the following ways

Not being able to go to work

Not being able to work as much or as effectively

Saving up sick hour to take off when experiencing symptoms

Feeling less comfortable with coworkers 
Table 1 (continued)

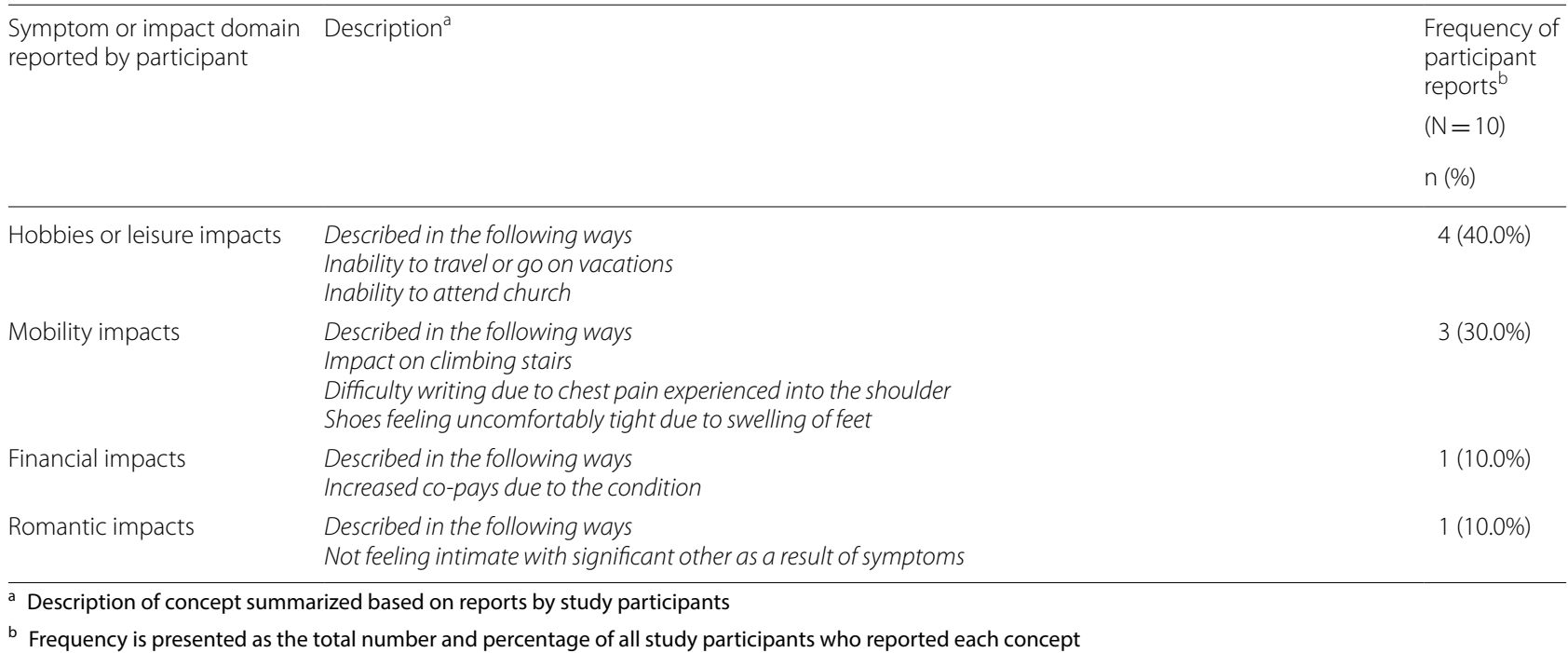

who were CS-dependent but not acutely symptomatic at baseline (CSD-RP; $n=9$ ). See Table 3 for the demographics and health characteristics of these two participant groups.

Scores from the PROMIS GH questionnaire items and domains were evaluated for each of the participant groups over time (baseline, end of TP, and end of EP). Figure 2 shows the baseline scores for the two domains of the PROMIS GH health questionnaire. For both the A-RP and CSD-RP groups, average scores for these domains are below the US normative average score of 50. Additionally, Table 4 shows a trend for improvement in some item and domain scores for both the A-RP and CSD-RP groups.

For participants in the A-RP group, increases in the average scores for items of the PROMIS GH questionnaire that assess general health, quality of life, and physical health indicate improvement over the study period, with large ESs. In addition, for the A-RP group, the average score of the PROMIS GH pain item shows the largest decrease over the study period, with a mean score of nearly 5 on the $0-10$ NRS at baseline, and less than 1 at end of $\mathrm{EP}(\mathrm{ES}=-2.69 ; 95 \% \mathrm{CI}=-3.66$ to -1.72$)$. At baseline, both the physical and mental domain scores $(\mathrm{GPH} / \mathrm{GMH})$ were lower than the normative average of 50 , but by end of TP mean scores for the GPH were above the US norm (and remained above at end of EP, with a large change $[\mathrm{ES}=1.48 ; 95 \% \mathrm{CI}=0.65$ to 2.23$)$, and mean scores for the GMH were at the US norm (and remained at the normative average at end of EP).

For participants in the CSD-RP group, there were modest increases (improvements) on the PROMIS $\mathrm{GH}$ items assessing mental health, social activities and relationships, social activities and roles, and emotional problems, with ESs indicative of medium changes (Cohen's d between 0.5 and 0.8). The average scores for the other items did not change. Similar to the A-RP group, average scores for the CSD-RP group were also below the US norm for both the GPH and GMH domains at baseline. For the GMH domain, average scores were at the normative average at the end of EP.

Figure 3 shows the change in the GPH and GMH domain scores over the study period, along with the trend between these HRQoL scores and patient-reported pericardial pain and serum marker of inflammation (CRP). For the A-RP group, pain scores and CRP levels decreased over the study period (change in pain scores from Baseline to end of EP was $4.6 \pm 1.82$ to $0.4 \pm 0.91$ $[\mathrm{ES}=-2.89,95 \% \mathrm{CI}=-3.90$ to -1.88$]$; change in CRP from Baseline to end of EP was $3.84 \pm 5.30$ to $0.24 \pm 0.36$ $[E S=-0.94,95 \% \mathrm{CI}=-1.89$ to -0.20$])$, while HRQoL scores increased. For the CSD-RP group, pericardial pain and CRP (low at baseline, as expected as these participants entered the trial while not in active recurrence) remained low over the course of the study even while tapering and discontinuing CS (change in pain scores from Baseline to end of EP was $1.4 \pm 1.51$ to $0.6 \pm 1.19$ $[\mathrm{ES}=-0.58 ; 95 \% \mathrm{CI}=-1.56$ to 0.39 ]; change in CRP from Baseline to end of EP was $0.19 \pm 0.11$ to $0.12 \pm 0.06$ $[\mathrm{ES}=-0.78 ; 95 \% \mathrm{CI}=-1.76$ to 0.21$]$ ), while $\mathrm{HRQoL}$ scores increase over time.

Please note that participants who completed the EP and were taking CS at Baseline (all participants in the CSD-RP [ $n=8]$, and $83.3 \%[n=5 / 6]$ of participants in the A-RP) were able to taper and/or discontinue using CS by the end of the EP (i.e., the end of the study) 


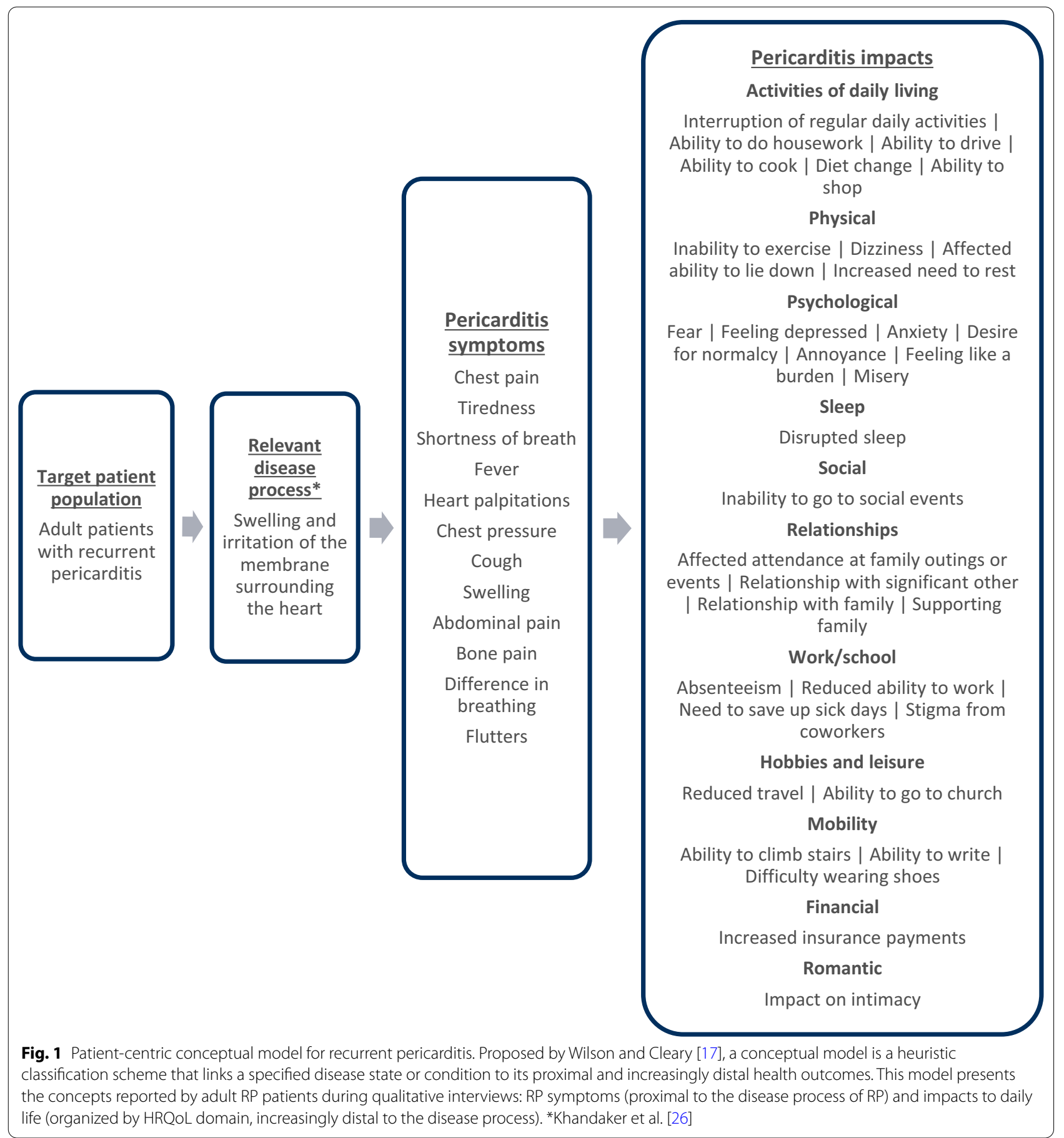

without recurrence or pericarditis symptomatology (e.g., patient-reported pericardial pain) or inflammation (e.g., elevated CRP level).

\section{Discussion}

While a substantial negative impact of RP on patients' HRQoL has traditionally been assumed, to our 


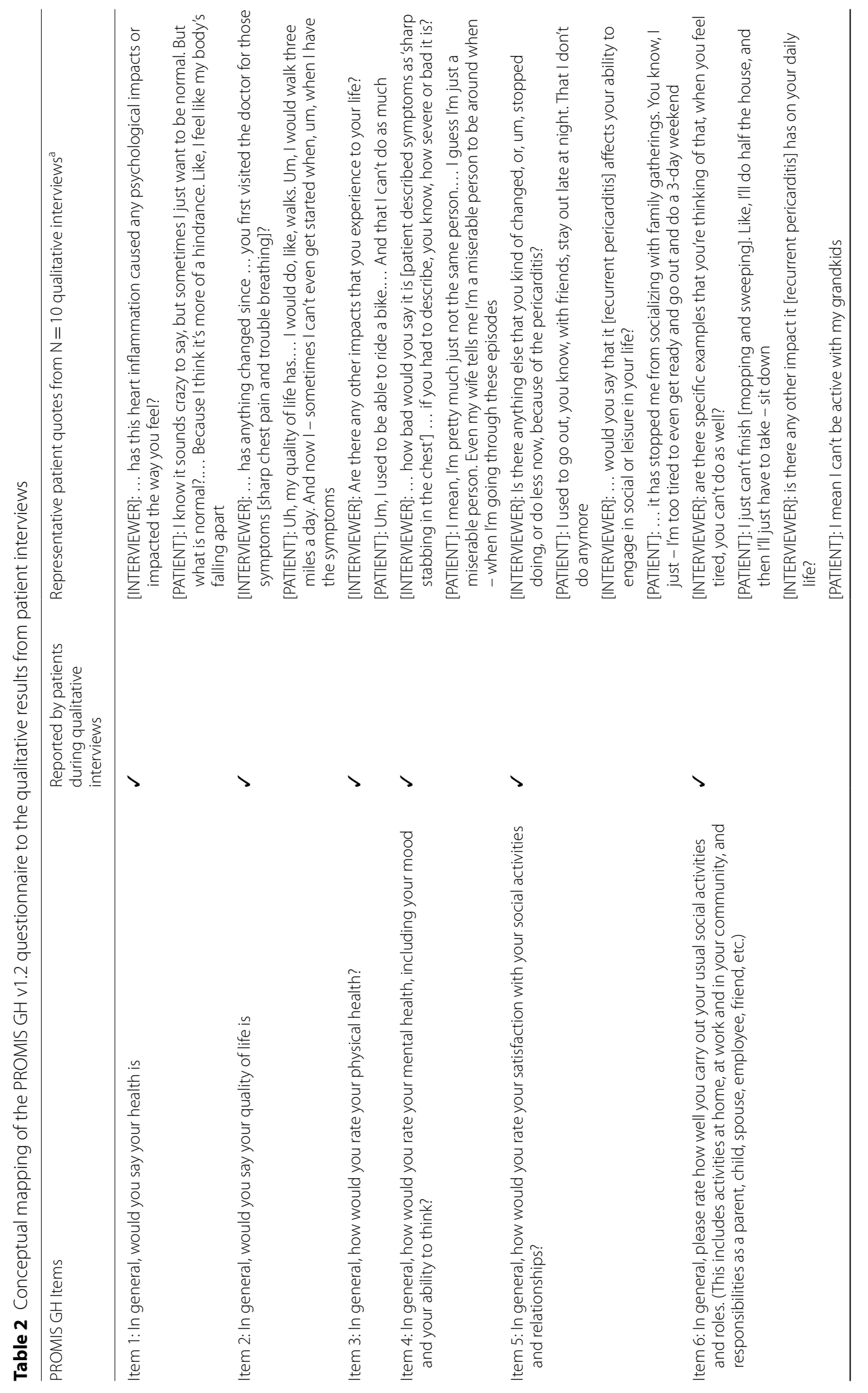




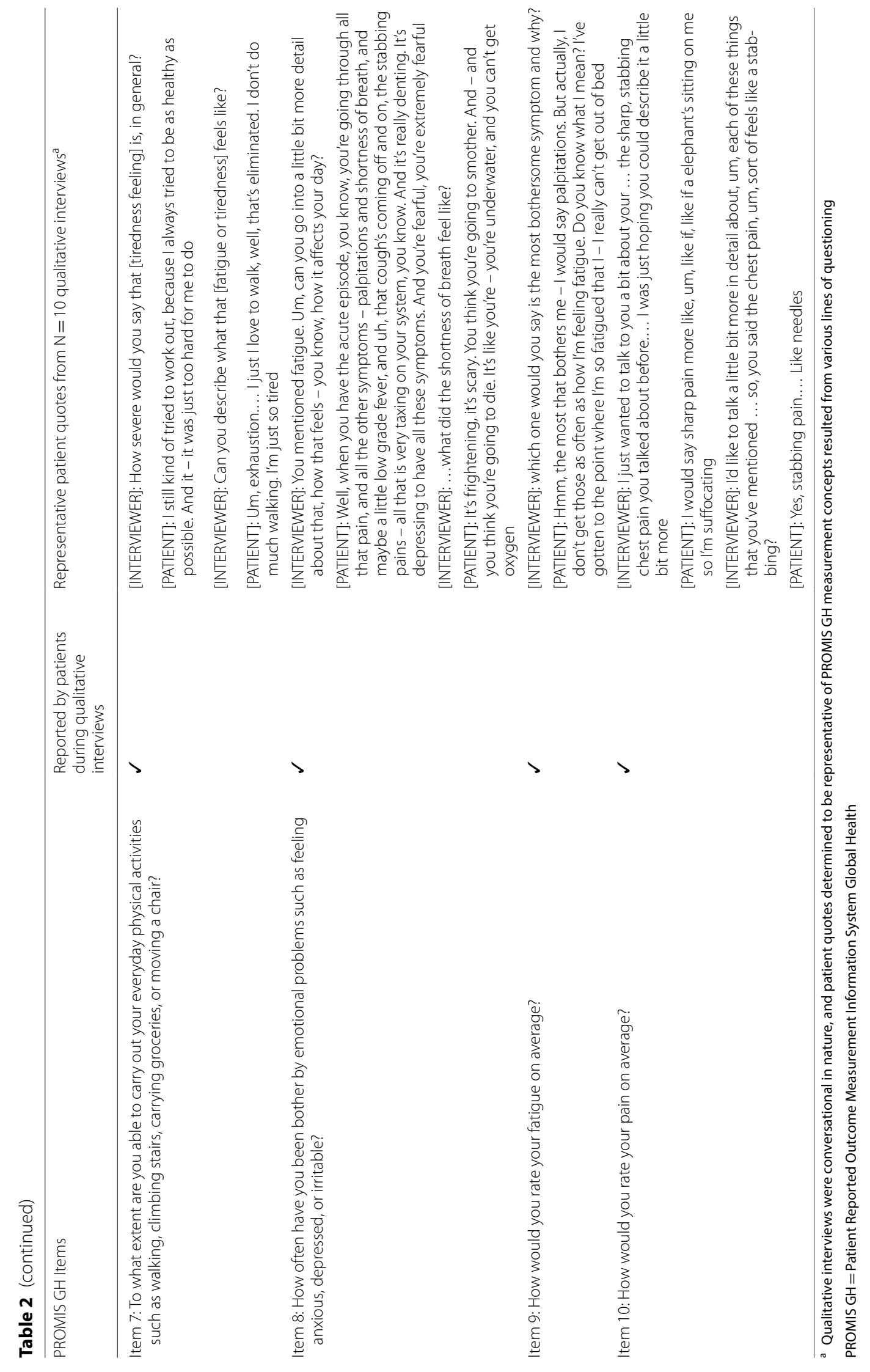


Table 3 Demographics and health characteristics of Phase 2 clinical trial sample

\begin{tabular}{|c|c|c|}
\hline \multirow[t]{2}{*}{ Characteristic } & Active recurrence (A-RP) & $\begin{array}{l}\text { Not symptomatic, } \\
\text { Corticosteroid-dependent } \\
\text { (CSD-RP) }\end{array}$ \\
\hline & $N=16$ & $N=9$ \\
\hline Age (years) (Mean $\pm S D$ [range]) & $39.8 \pm 10.52(26-58)$ & $48.2 \pm 8.56(36-62)$ \\
\hline Gender (\% female [n]) & $75.0 \%(n=12)$ & $33.3 \%(n=3)$ \\
\hline Race (\% white $[n])$ & $81.3 \%(n=13)$ & $100 \%(n=9)$ \\
\hline BMI $\left(\mathrm{kg} / \mathrm{m}^{2}\right)($ Mean $\pm S D$ [range] $)$ & $31.99 \pm 7.51(23.4-52.7)$ & $28.97 \pm 4.68(22.5-34.3)$ \\
\hline Duration of disease (years) (Mean \pm SD [range]) & $2.6 \pm 2.13(0.2-7.9)$ & $1.4 \pm 0.97(0.6-3.4)$ \\
\hline Number of prior recurrences (median, [range]) & $2(1-8)$ & $3(2-5)$ \\
\hline Baseline NRS Pain Rating 0-10 (Mean \pm SD [range]) & $4.6 \pm 1.82(2-8)$ & $1.4 \pm 1.51(0-5)$ \\
\hline Baseline CRP values (mg/dL) (Mean \pm SD [range]) & $3.8 \pm 5.30(0.09-19.84)$ & $0.19 \pm 0.11(0.05-0.36)$ \\
\hline \multicolumn{3}{|l|}{ Concomitant medications at baseline } \\
\hline Aspirin (n [\%]) & $0(0 \%)$ & $2(22.2 \%)$ \\
\hline NSAID (n [\%]) & $7(43.8 \%)$ & $5(55.6 \%)$ \\
\hline Colchicine (n [\%]) & $12(75.0 \%)$ & $8(88.9 \%)$ \\
\hline CS (n [\%]) & $6(37.5 \%)^{\mathrm{a}}$ & $9(100.0 \%)^{b}$ \\
\hline
\end{tabular}

$\mathrm{BMI}=$ body mass index; $\mathrm{CRP}=\mathrm{c}$-reactive protein; $\mathrm{CS}=$ corticosteroids; $\mathrm{EP}=$ extension period; $\mathrm{NRS}=$ numeric rating scale; $\mathrm{NSAID}=$ nonsteroidal anti-inflammatory drug; $\mathrm{SD}=$ standard deviation

a $4 / 6(66.7 \%)$ discontinued CS and 1/6 (16.7\%) tapered CS by end of EP; $1 / 6$ (16.7\%) did not enter EP

b $7 / 9(77.8 \%)$ discontinued CS and 1/9 (11.1\%) tapered CS by end of EP; $1 / 9$ (11.1\%) did not enter EP

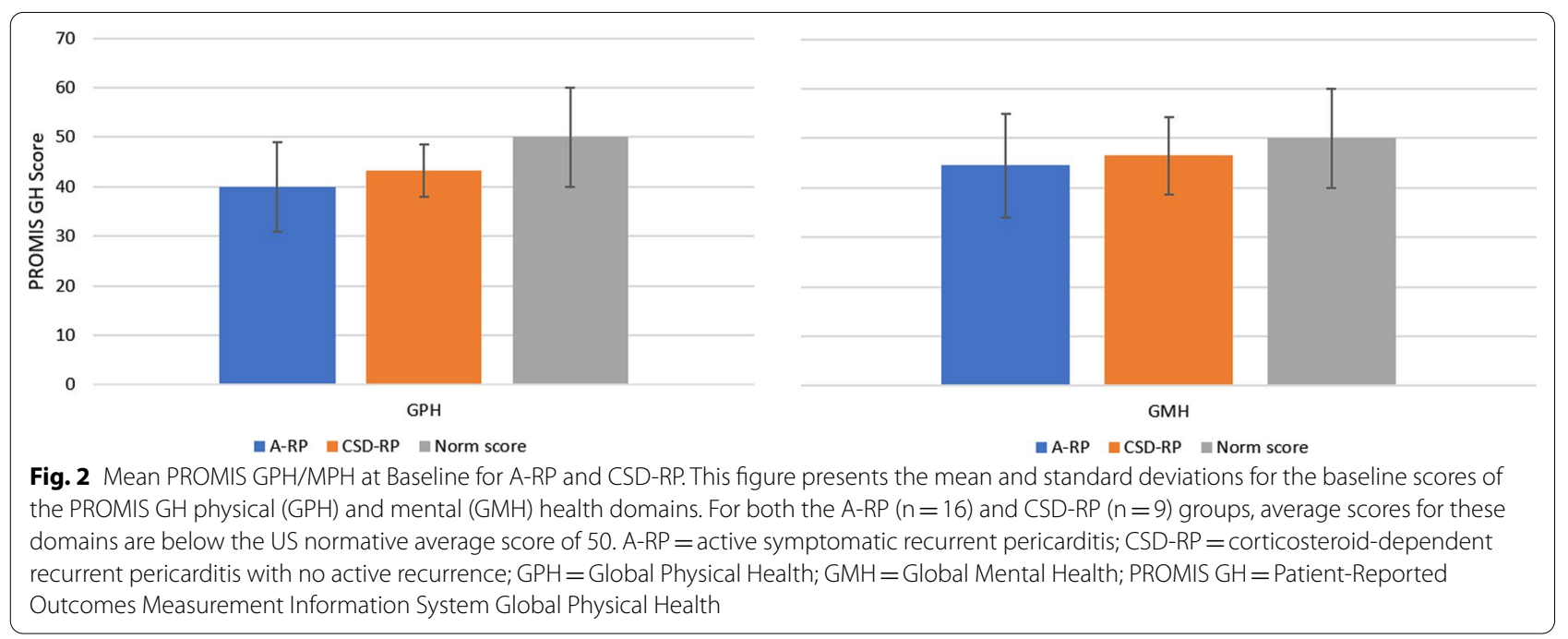

knowledge this is the first analysis using qualitative and quantitative methods to explore the ways that symptoms of pericarditis recurrence impact patients' quality of life. The results from the baseline timepoint of the Phase 2 clinical trial align with the assumption of patients' decreased HRQoL, showing that scores on the GMH and GPH of the PROMIS GH questionnaire were on average lower than normative scores for both the A-RP and CSD-RP groups. In addition, the improvement in HRQoL scores over the course of the study tracks with improvements in patient-reported pericardial pain and CRP levels for the A-RP group, and with the tapering and discontinuing of CS for the CSD-RP group while pericardial pain and CRP remained stable and low while on rilonacept treatment. For the A-RP group, the magnitude of change between Baseline and End of EP was large for the PROMIS GH physical health, pain, and quality of life items, and the GPH domain (Cohen's $d \geq 0.80$ ). In contrast, for the CSD-RP group, the changes between Baseline and End of EP for the mental health item and the 
Table 4 PROMIS GH item and domain scores over time (mean \pm SD), by participant group

\begin{tabular}{|c|c|c|c|c|c|c|c|c|}
\hline \multirow{2}{*}{$\begin{array}{l}\text { PROMIS GH } \\
\text { item/ domain }\end{array}$} & \multicolumn{4}{|c|}{ Active recurrence (A-RP) } & \multicolumn{4}{|c|}{ Not symptomatic, Corticosteroid-dependent (CSD-RP) } \\
\hline & $\begin{array}{l}\text { Baseline } \\
(n=16)\end{array}$ & $\begin{array}{l}\text { End of TP visit } \\
(n=15)\end{array}$ & $\begin{array}{l}\text { End of EP visit } \\
(n=15)\end{array}$ & $\mathrm{ES}(95 \% \mathrm{Cl})^{\mathrm{b}}$ & $\begin{array}{l}\text { Baseline } \\
(n=7)\end{array}$ & $\begin{array}{l}\text { End of TP visit } \\
(n=9)\end{array}$ & $\begin{array}{l}\text { End of EP visit } \\
(n=8)\end{array}$ & ES $(95 \% \mathrm{Cl})^{\mathrm{b}}$ \\
\hline $\mathrm{GPH}$ & $39.94 \pm 8.94$ & $51.35 \pm 7.96$ & $51.32 \pm 6.56$ & $\begin{array}{l}1.44 \text { (0.65 to } \\
2.23)\end{array}$ & $43.30 \pm 5.31$ & $45.09 \pm 4.06$ & $46.81 \pm 9.27$ & $\begin{array}{l}0.46(-0.57 \text { to } \\
1.48)\end{array}$ \\
\hline $\begin{array}{l}\text { Item 3: physi- } \\
\text { cal health }\end{array}$ & $2.6 \pm 0.96$ & $3.2 \pm 1.01$ & $3.5 \pm 0.83$ & $\begin{array}{l}\mathbf{1 . 0 0}(0.25 \text { to } \\
1.75)\end{array}$ & $2.8 \pm 0.46$ & $3.1 \pm 0.33$ & $3.0 \pm 0.93$ & $\begin{array}{l}0.27(-0.75 \text { to } \\
1.29)\end{array}$ \\
\hline $\begin{array}{l}\text { Item 7: physi- } \\
\text { cal activities }\end{array}$ & $3.3 \pm 1.39$ & $4.4 \pm 1.06$ & $4.1 \pm 1.03$ & $\begin{array}{l}0.65(-0.07 \text { to } \\
1.37)\end{array}$ & $3.4 \pm 0.74$ & $3.3 \pm 0.87$ & $3.8 \pm 1.04$ & $\begin{array}{l}0.44(-0.59 \text { to } \\
1.46)\end{array}$ \\
\hline Item 9: fatigue & $3.1 \pm 0.96$ & $3.7 \pm 0.49$ & $3.7 \pm 0.82$ & $\begin{array}{l}0.67(-0.05 \text { to } \\
1.39)\end{array}$ & $3.1 \pm 0.69$ & $3.2 \pm 0.44$ & $3.4 \pm 1.06$ & $\begin{array}{l}0.33(-0.69 \text { to } \\
1.35)\end{array}$ \\
\hline Item 10: pain & $4.8 \pm 1.88$ & $0.6 \pm 1.18$ & $0.5 \pm 1.13$ & $\begin{array}{l}\mathbf{- 2 . 6 9}(-3.66 \text { to } \\
-1.72)\end{array}$ & $1.7 \pm 1.60$ & $1.0 \pm 1.32$ & $1.4 \pm 2.50$ & $\begin{array}{l}-0.14(-1.16 \text { to } \\
0.87)\end{array}$ \\
\hline GMH & $44.50 \pm 10.48$ & $50.13 \pm 11.33$ & $50.54 \pm 11.00$ & $\begin{array}{l}0.56(-0.16 \text { to } \\
1.28)\end{array}$ & $46.49 \pm 7.77$ & $47.91 \pm 5.51$ & $50.66 \pm 6.30$ & $\begin{array}{l}0.59(-0.44 \text { to } \\
1.63)\end{array}$ \\
\hline $\begin{array}{l}\text { Item 2: quality } \\
\text { of life }\end{array}$ & $3.0 \pm 1.03$ & $3.6 \pm 1.06$ & $4.0 \pm 1.00$ & $\begin{array}{l}\mathbf{0 . 9 8}(0.24 \text { to } \\
1.73)\end{array}$ & $3.3 \pm 1.04$ & $3.6 \pm 0.73$ & $3.4 \pm 0.74$ & $\begin{array}{l}0.11(-0.90 \text { to } \\
1.13)\end{array}$ \\
\hline $\begin{array}{l}\text { Item 4: mental } \\
\text { health }\end{array}$ & $3.3 \pm 1.13$ & $3.7 \pm 1.23$ & $3.6 \pm 1.12$ & $\begin{array}{l}0.27(-0.44 \text { to } \\
0.97)\end{array}$ & $3.4 \pm 0.74$ & $3.6 \pm 0.73$ & $3.9 \pm 0.83$ & $\begin{array}{l}0.63(-0.41 \text { to } \\
1.67)\end{array}$ \\
\hline $\begin{array}{l}\text { Item 5: social } \\
\text { activities and } \\
\text { relation- } \\
\text { ships }\end{array}$ & $3.1 \pm 1.34$ & $3.7 \pm 1.18$ & $3.6 \pm 1.12$ & $\begin{array}{l}0.40(-0.31 \text { to } \\
1.12)\end{array}$ & $3.1 \pm 0.83$ & $3.3 \pm 0.50$ & $3.6 \pm 0.92$ & $\begin{array}{l}0.57(-0.47 \text { to } \\
1.60)\end{array}$ \\
\hline $\begin{array}{l}\text { Item 8: } \\
\text { emotional } \\
\text { problems }\end{array}$ & $3.1 \pm 1.41$ & $3.5 \pm 1.36$ & $3.4 \pm 1.12$ & $\begin{array}{l}0.23(-0.47 \text { to } \\
0.94)\end{array}$ & $3.4 \pm 0.98$ & $3.3 \pm 0.71$ & $4.0 \pm 0.53$ & $\begin{array}{l}0.78(-0.27 \text { to } \\
1.83)\end{array}$ \\
\hline \multicolumn{9}{|c|}{ Items that are not included in above domains } \\
\hline $\begin{array}{l}\text { Item 1: general } \\
\text { health }\end{array}$ & $2.9 \pm 0.72$ & $3.5 \pm 0.83$ & $3.6 \pm 0.91$ & $\begin{array}{l}\mathbf{0 . 8 2}(0.12 \text { to } \\
1.59)\end{array}$ & $2.9 \pm 0.64$ & $3.1 \pm 0.33$ & $3.1 \pm 0.64$ & $\begin{array}{l}0.31(-0.71 \text { to } \\
1.33)\end{array}$ \\
\hline $\begin{array}{l}\text { Item } 6 \text { : social } \\
\text { activities and } \\
\text { roles }\end{array}$ & $3.1 \pm 1.09$ & $3.5 \pm 1.25$ & $3.5 \pm 1.13$ & $\begin{array}{l}0.36(-0.35 \text { to } \\
1.07)\end{array}$ & $2.9 \pm 0.99$ & $3.1 \pm 0.93$ & $3.5 \pm 0.93$ & $\begin{array}{l}0.63(-0.41 \text { to } \\
1.67)\end{array}$ \\
\hline
\end{tabular}

$\mathrm{Cl}=$ confidence interval; $\mathrm{EP}=$ extension period; $\mathrm{ES}=$ effect size; $\mathrm{GMH}=$ Global Mental Health; GPH = Global Physical Health; PROMIS GH = Patient Reported Outcome Measurement Information System Global Health; TP = treatment period

a For Items 1-9, scores range from 1 to 5 with higher scores indicating improvement, and for Item 10, scores range from 0 to 10 with lower scores indicating improvement. Scoring for Item 10 is adjusted when calculating the GPH. To calculate the GMH and GPH domain scores, raw scores are converted to standardized T scores, with a normative mean of 50 and standard deviation of 10

b ES is calculated from Baseline and End of EP Visit; Bolded values are large $(\geq 0.80)$

GMH domain (Cohen's d 0.50 to $<0.80$ ), while not large (which was expected, given the absence of acute pericarditis recurrences) were not insubstantial, likely due to the tapering and discontinuation of corticosteroids. Future studies should investigator further the impact of CS use on HRQoL, and how discontinuation of CS for patients with RP on targeted therapy impacts physical and emotional HRQoL. Taken together, these results show that RP negatively impacts patients' quality of life physically and emotionally, and that improvements in quality of life may be associated with improvement in disease symptomatology and a decrease in pericardial inflammation, in particular, while patients receive targeted treatment.

Furthermore, results from qualitative interviews, where adults with RP spoke about the unpredictable nature of the condition, supported that pericarditis recurrences impact patient physical and mental health. Specifically, using the qualitative data, a conceptual model of RP was developed, and HRQoL concepts included in the concept model (e.g., ability to carry out daily activities, impacts on mood, and limitation on social activities) were mapped against the ten items of the PROMIS GH v1.2 questionnaire (included in the Phase 2 clinical trial of rilonacept), which demonstrate that this questionnaire is assessing concepts that are relevant to adults with RP.

Limitations include the small sample sizes for both the qualitative interviews and the clinical trial, the singleactive-arm design of the clinical trial, and the relatively short duration (24 weeks) of the clinical trial compared to the overall duration of this chronic disease. In addition, 


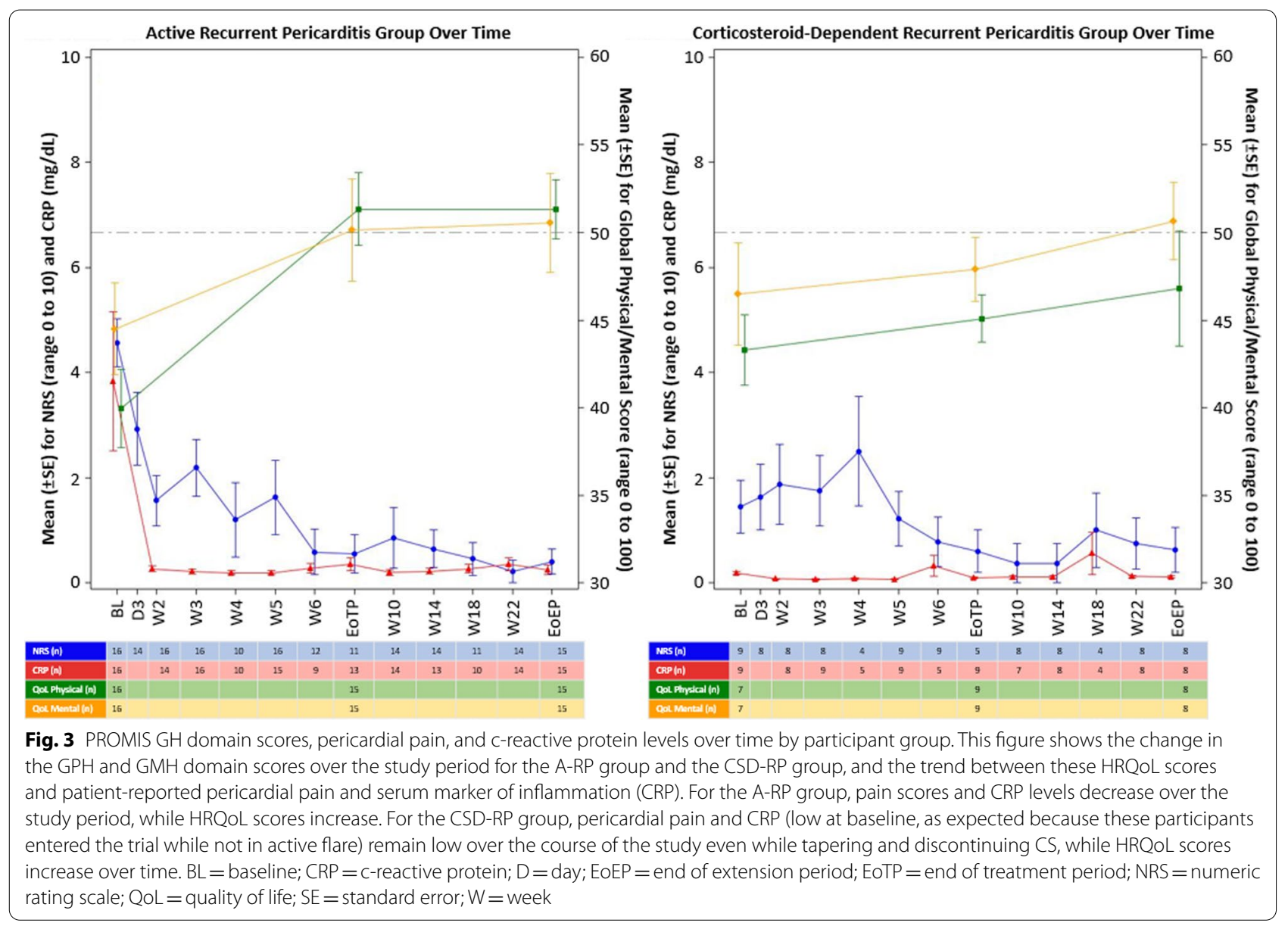

while the inclusion criteria for the qualitative interview study were intended to be similar to those of the clinical trial, they were less restrictive (i.e., adults interviewed did not experience as many recurrences as the participants in the clinical trial). Nevertheless, these results provide preliminary support for the importance of including a multidimensional assessment of HRQoL for future clinical research of RP. It is also important to consider that some HRQoL impacts may be dependent on age and gender, therefore, given the age range of the participants who completed the qualitative interviews and the Phase 2 clinical trial, the resulting conceptual model should be considered representative of adult RP.

Strengths include leveraging qualitative results to support the importance of the item- and domain-level scores of the PROMIS GH v1.2 questionnaire to adults with RP. The representative patient quotes help contextualize how participants may be interpreting each item of the PROMIS GH questionnaire. In addition, the means for the PROMIS GH domain scores at baseline in the rilonacept clinical trial provide evidence of the impact RP has on patients' HRQoL, as they are lower compared to population norm scores. These findings are consistent with other clinical studies reporting lower PROMIS GH questionnaire scores and associated impacts in physical, mental, and social domains in cardiac and vascular populations [23-25]. The increase in both the GPH and GMH scores over the course of the study for both the A-RP and CSD-RP, in conjunction with improvements and/ or stable pericardial pain scores and CRP levels, shows that HRQoL scores may also be responsive to treatment as the patient's condition improves, particularly when on a treatment that addresses IL-1 driven pericardial inflammation.

\section{Conclusions}

Given the anxiety associated with the unpredictability of recurrences and the exercise restrictions that patients are expected to adhere to following a diagnosis of RP, it is important to evaluate both emotional and physical impacts of the condition. As more clinical trials move to incorporate patient-centric outcomes to evaluate treatments not only in terms of resolution of a physiological indicator of disease but also to ensure 
that patients feel and function better, future clinical trials of adults with RP should include HRQoL PRO questionnaires. In addition, future studies should explicitly examine the effect of concomitant medications, including CS, and their independent impact on patient HRQoL.

The results of this pilot study may suggest a signal of a positive impact of rilonacept on clinical outcome measures and improvements in patient HRQoL over the study time period which tracked with improvements in pericardial pain and inflammation. For those participants in the CSD-RP group, who were weaning off CS while taking rilonacept, patient-reported pericardial pain and CRP levels were stable, while HRQoL scores improved over the course of the study, without recurrences. With the approval of rilonacept, appropriate patients with RP may now have a CS-sparing treatment alternative that not only reduces pain and inflammation but also reduces the risk of recurrence while improving HRQoL.

\begin{abstract}
Abbreviations
A-RP: Active recurrence of pericarditis at baseline; CRP: C-reactive protein; CS: Corticosteroids; CSD-RP: Dependent on corticosteroids at baseline; EP: Extension period; GMH: Global mental health; GPH: Global physical health; HRQoL: Health-related quality of life; IRB: Independent review board; NRS: Numeric rating scale; NSAIDs: Nonsteroidal anti-inflammatory drugs; PRO: Patientreported outcome; PROMIS GH: Patient Reported Outcome Measurement Information System Global Health; RP: Recurrent pericarditis; SC: Subcutaneous; TP: Treatment period.
\end{abstract}

\begin{abstract}
Acknowledgements
All MRI findings were analyzed by the Imaging Core Laboratory C5Research (Cleveland Clinic). Sharon Crugnale and Larisa Collins were employed by Kiniksa Pharmaceuticals Corp. as clinical operations managers at the time the study was conducted; Kasia Warchol, Steven Chang, Cory Burke, Heather Cong, Randy Perrin and Jeannie Celiberti, all employees of Kiniksa Pharmaceuticals Corp., contributed to data collection and/or analysis. Kristi Wort, project manager at TCTM, contributed to the design of the study and its data collection. Scott Mellis, Regeneron Pharmaceuticals, Inc. employee, offered greatly valuable insights to the program. Eugene Luau and Bruce Green, from Model Answers performed the pharmacokinetic analyses and interpretation. Monica Brova and Sylvia Su contributed to the design of the qualitative study and its data collection and analysis. We want to thank all the patients and their caregivers, the study coordinators, the investigators, and all the investigative site personnel who participated in this study; medical writing assistance was provided by Emmanuelle Hugentobler, a Kiniksa Pharmaceuticals Corp. employee, and in part by Peloton Advantage, an OPEN Health company, funded by Kiniksa Pharmaceuticals Ltd.
\end{abstract}

\section{Authors' contributions}

Study design: JFP, AB, AK, PC, LLK (qualitative interview study), BK (qualitative interview study). Study investigator: AK, MLW, DL, PC, SAL, AA, AE, SN. Enrolled patients: AK, MLV, DL, PC, SAL, AA, AE, SN. Collection and assembly of data: $A B, F F, A K, P C, S A L, L L K$ (qualitative interview study), BK (qualitative interview study). Data analysis: JFP, AB, FF, AK, PC, LLK (qualitative interview study), BK (qualitative interview study). Data interpretation: All authors. Manuscript preparation: JFP, MM, LLK, BK. Manuscript review and revisions: All authors. Final approval of manuscript: All authors. Independent data access and analysis: Throughout the writing of this paper, all authors had the ability to query any aspect of the data either directly or through independent analysis and retain this ability until publication. Anna Beutler, John Paolini, and Fang Fang had full access to all the data in the study and take responsibility for its integrity and the data analysis. All authors read and approved the final manuscript.
Funding

This study was funded by Kiniksa Pharmaceuticals, Ltd.

Availability of data and materials

Data will be made available upon reasonable request.

\section{Declarations}

Ethics approval and consent to participate

Ethics approval for the qualitative interviews was granted by Sterling IRB under IRB ID\# 6406. Ethics approval for the Phase 2 study was granted by the central IRB Aspire under IRB ID\# KPL-914-C001 as well as by site-specific central and local IRBs, including Chesapeake IRB, Cleveland Clinic Foundation IRB, Mayo Clinic IRB, University of Vermont Research Protections Office Committee on Human Research in the Medical Sciences, and Western IRB.

\section{Consent for publication}

Not applicable.

\section{Competing interests}

David Lin: None. Allan Klein: Research grant, scientific advisory board Kiniksa Pharmaceuticals, Ltd., advisory board Swedish Orphan Biovitrum AB, advisory board Pfizer, Inc., modest. David Cella: Consultant for Kiniksa Pharmaceuticals, Ltd., modest. Anna Beutler: Kiniksa Pharmaceuticals, Ltd. consultant. Fang Fang: Kiniksa Pharmaceuticals, Corp. employee. Matt Magestro: Kiniksa Pharmaceuticals, Corp. employee. Paul Cremer: Advisory board Swedish Orphan Biovitrum AB, advisory board Kiniksa Pharmaceuticals, Ltd., modest. Martin M. LeWinter: One seminar for Kiniksa Pharmaceuticals, Ltd., modest. Sushil Allen Luis: Advisory board member for Kiniksa Pharmaceuticals, Ltd., modest. Consultant and advisory board member for Swedish Orphan Biovitrum AB, significant. Antonio Abbate: Research grants from Kiniksa Pharmaceuticals, Ltd., Swedish Orphan Biovitrum AB, Olatec Therapeutics LLC, Serpin Pharma, LLC; consultant fees: Kiniksa Pharmaceuticals, Ltd., Olatec Therapeutics LLC, Serpin Pharma, LLC, Merck \& Co., Inc., modest. Andrew Ertel: None. Leighann Litcher-Kelly: Employed by Adelphi Values, which received funding from Kiniksa Pharmaceuticals, Ltd. for PRO work in pericarditis. Brittany Klooster: Employed by Adelphi Values, which received funding from Kiniksa Pharmaceuticals, Ltd. for PRO work in pericarditis. John F. Paolini: Kiniksa Pharmaceuticals, Corp. Employee.

\section{Author details}

${ }^{1}$ Abbott Northwestern's Heart Hospital, Minneapolis Heart Institute, 800 East 28th Street, 2nd Floor, Minneapolis, MN 55407, USA. ${ }^{2}$ Cleveland Clinic, Cleveland, OH, USA. ${ }^{3}$ Northwestern University, Evanston, IL, USA. ${ }^{4}$ Kiniksa Pharmaceuticals Corp., 100 Hayden Avenue, Lexington, MA 02421, USA. ${ }^{5}$ University of Vermont Medical Center, Burlington, VT, USA. ${ }^{6}$ Mayo Clinic, Rochester, MN, USA. ${ }^{7}$ Virginia Commonwealth University, Richmond, VA, USA. ${ }^{8}$ Medstar Heart and Vascular Institute, Washington, DC, USA. ${ }^{9}$ Adelphi Values Patient-Centered Outcomes, Boston, MA, USA.

Received: 4 February 2021 Accepted: 12 April 2021

Published online: 21 April 2021

\section{References}

1. Adler Y, Charron P, Imazio M, Badano L, Barón-Esquivias G, Bogaert J, et al. 2015 ESC Guidelines for the diagnosis and management of pericardial diseases. The Task Force for the Diagnosis and Management of Pericardial Diseases of the European Society of Cardiology (ESC). Endorsed by: The European Association for Cardio-Thoracic Surgery (EACTS). Eur Heart J. 2015;36(42):2921-64.

2. Brucato A, Imazio M, Cremer PC, Adler Y, Maisch B, Lazaros G, et al. Recurrent pericarditis: still idiopathic? The pros and cons of a well-honoured term. Intern Emerg Med. 2018;13(6):839-44.

3. Perrone A, Castrovilli A, Piazzolla G, Savino S, D'Introno A, Sabbà C. Corticosteroids for acute and recurrent idiopathic pericarditis: unexpected evidences. Cardiol Res Pract. 2019;2019:1348364. 
4. Buckley LF, Viscusi MM, Van Tassell BW, Abbate A. Interleukin-1 blockade for the treatment of pericarditis. Eur Heart J Cardiovasc Pharmacother. 2018;4(1):46-53.

5. Klein AL, Lin D, Cremer PC, Nasir S, Luis SA, Abbate A, et al. Efficacy and safety of rilonacept for recurrent pericarditis: results from a phase II clinical trial. Heart. 2020

6. Klein AL, Imazio M, Cremer P, Brucato A, Abbate A, Fang F, et al. Phase 3 trial of interleukin-1 trap rilonacept in recurrent pericarditis. N Engl J Med. 2021:384(1):31-41.

7. Imazio M, Gribaudo E, Gaita F. Recurrent Pericarditis. Prog Cardiovasc Dis. 2017:59(4):360-8.

8. Lotan D, Wasserstrum Y, Fardman A, Kogan M, Adler Y. Usefulness of novel immunotherapeutic strategies for idiopathic recurrent pericarditis. Am J Cardiol. 2016;117(5):861-6.

9. Soler-Soler J, Sagristà-Sauleda J, Permanyer-Miralda G. Relapsing pericarditis. Heart. 2004;90(11):1364.

10. Raatikka M, Pelkonen PM, Karjalainen J, Jokinen EV. Recurrent pericarditis in children and adolescents: report of 15 cases. J Am Coll Cardiol. 2003:42(4):759-64.

11. LeWinter M, Kontzias A, Lin D, Cella D, DerSarkissian M, Zhou M, et al. Burden of recurrent pericarditis on health-related quality of life. Am J Cardiol. 2021;141:113-9.

12. Lin D, Klein A, Cella D, Beutler A, Fang F, Magestro M, et al. Health-Related Quality Of Life In Patients With Recurrent Pericarditis: Results From A Phase 2 Study Of Rilonacept. Quality of Care and Outcomes Research Scientific Sessions; 2020

13. Imazio M, Brucato A, Cumetti D, Brambilla G, Demichelis B, Ferro S, et al. Corticosteroids for recurrent pericarditis. Circulation. 2008;118(6):667-71.

14. Assolari A, Maestroni S, Cumetti D, Valenti A, Parisi F, Brucato A. Clinical management and therapy of idiopathic recurrent pericarditis. Clin Manag Issues. 2018;12

15. Judson MA, Chaudhry H, Louis A, Lee K, Yucel R. The effect of corticosteroids on quality of life in a sarcoidosis clinic: the results of a propensity analysis. Respir Med. 2015;109(4):526-31.

16. McDonald CM, Henricson EK, Abresch RT, Duong T, Joyce NC, Hu F, et al. Long-term effects of glucocorticoids on function, quality of life, and survival in patients with Duchenne muscular dystrophy: a prospective cohort study. Lancet. 2018;391(10119):451-61.
17. Wilson IB, Cleary PD. Linking clinical variables with health-related quality of life. A conceptual model of patient outcomes. JAMA. 1995:273(1):59-65.

18. Dworkin RH, Turk DC, Farrar JT, Haythornthwaite JA, Jensen MP, Katz NP, et al. Core outcome measures for chronic pain clinical trials: IMMPACT recommendations. Pain. 2005;113(1-2):9-19.

19. Hawker GA, Mian S, Kendzerska T, French M. Measures of adult pain: Visual Analog Scale for Pain (VAS Pain), Numeric Rating Scale for Pain (NRS Pain), McGill Pain Questionnaire (MPQ), Short-Form McGill Pain Questionnaire (SF-MPQ), Chronic Pain Grade Scale (CPGS), Short Form-36 Bodily Pain Scale (SF-36 BPS), and Measure of Intermittent and Constant Osteoarthritis Pain (ICOAP). Arthritis Care Res (Hoboken). 2011;63(Suppl 11):S240-52.

20. Mannion AF, Balague F, Pellise F, Cedraschi C. Pain measurement in patients with low back pain. NatClin PractRheumatol. 2007;3(11):610-8.

21. Hays RD, Bjorner JB, Revicki DA, Spritzer KL, Cella D. Development of physical and mental health summary scores from the patient-reported outcomes measurement information system (PROMIS) global items. QualLife Res. 2009;18(7):873-80.

22. Cohen J. Statistical Power Analysis for the Behavioral Sciences. Hillsdale: Lawrence Earlbaum Associates; 1988. p. 1988.

23. Charles E, Mehaffey J, Hawkins R, Burks S, McMurry T, Yarboro L, et al. Meaningful patient-centered outcomes 1 year following cardiac surgery. Ann Surg. 2019:1.

24. Lam KH, Kwa VIH. Validity of the PROMIS-10 Global Health assessed by telephone and on paper in minor stroke and transient ischaemic attack in the Netherlands. BMJ Open. 2018;8(7):e019919-e.

25. Ahmad FS, Kallen MA, Schifferdecker KE, Carluzzo KL, Yount SE, Gelow JM, et al. Development and initial validation of the PROMIS ${ }^{\circledR}$-plus-HF profile measure. Circ Heart Fail. 2019;12(6):e005751.

26. Khandaker MH, Espinosa RE, Nishimura RA, Sinak LJ, Hayes SN, Melduni $\mathrm{RM}$, et al. Pericardial disease: diagnosis and management. Mayo Clin Proc. 2010;85(6):572-93.

\section{Publisher's Note}

Springer Nature remains neutral with regard to jurisdictional claims in published maps and institutional affiliations.
Ready to submit your research? Choose BMC and benefit from:

- fast, convenient online submission

- thorough peer review by experienced researchers in your field

- rapid publication on acceptance

- support for research data, including large and complex data types

- gold Open Access which fosters wider collaboration and increased citations

- maximum visibility for your research: over $100 \mathrm{M}$ website views per year

At BMC, research is always in progress.

Learn more biomedcentral.com/submissions 